\title{
Caracterização das propriedades tecnológicas, físico-químicas e sensoriais de geleias
}

\section{de frutas tropicais}

\author{
Characterization of technological, physicochemical and sensory properties of tropical fruit jellies \\ Caracterización de las propiedades tecnológicas, fisicoquímicas y sensoriales de las jaleas de frutas
}

tropicales

Recebido: 22/07/2021 | Revisado: 30/07/2021 | Aceito: 31/07/2021 | Publicado: 06/08/2021

\author{
Jéssica Lima de Morais \\ ORCID: https://orcid.org/0000-0002-1511-9114 \\ Universidade Federal da Paraíba, Brasil \\ E-mail: jessicamorais-pb@hotmail.com \\ Mayara Gabrielly Germano de Araújo \\ ORCID: https://orcid.org/0000-0002-2966-7875 \\ Universidade Federal do Rio Grande do Norte, Brasil \\ E-mail: mayaragabrielly.c@gmail.com \\ Edson Douglas Silva Pontes \\ ORCID: https://orcid.org/0000-0002-7642-9466 \\ Universidade Federal de Campina Grande, Brasil \\ E-mail: edsondspontes@gmail.com \\ Vanessa Bordin Viera \\ ORCID: https://orcid.org/0000-0003-4979-4510 \\ Universidade Federal de Campina Grande, Brasil \\ E-mail: vanessa.bordinviera@ gmail.com \\ Rossana Maria Feitosa Figueiredo \\ ORCID: https://orcid.org/0000-0002-6187-5826 \\ Universidade Federal de Campina Grande, Brasil \\ E-mail: rossanamff@gmail.com \\ Maria Elieidy Gomes de Oliveira \\ ORCID: https://orcid.org/0000-0001-9870-9381 \\ Universidade Federal da Paraíba, Brasil \\ E-mail: elieidynutri@yahoo.com.br
}

\begin{abstract}
Resumo
Neste estudo objetivou-se desenvolver e caracterizar três formulações de geleia, utilizando morango e acerola de forma isolada e mista. Foram elaboradas três formulações: GA - geleia de acerola; GM - geleia de morango; GAM geleia mista de acerola e morango. Para a caracterização das amostras foram realizadas análises de determinação de $\mathrm{pH},{ }^{\circ}$ Brix, acidez total titulável (ATT) viscosidade, umidade, açucares totais (AT), glicose, sacarose, atividade antioxidante (FRAP e ABTS) e do conteúdo total de fenólicos e flavonoides. Além disto, as amostras foram submetidas a teste de aceitação sensorial com 100 provadores não treinados para verificar a opinião dos mesmos em relação aos atributos aparência, cor, aroma, sabor, consistência, avaliação global e intenção de compra. Os resultados demonstraram que as geleias apresentaram teores de sólidos solúveis ( ${ }^{\circ}$ Brix) variando entre $56-68,17{ }^{\circ}$ Brix, os quais foram suficientes para a formação do gel. As geleias não apresentaram diferença significativa $(p \geq 0,05)$ apenas para os parâmetros de pH, AT e sacarose. A viscosidade das geleias diminuiuram de acordo com o aumento da temperatura. Neste estudo percebeu-se que a mistura das duas frutas aumentou a aceitação sensoriais dos atributos avaliados; todavia, em termos de funcionalidade e aspectos tecnológicos, a geleia feita apenas com acerola apresentou maiores atividades antioxidantes e viscosidade em temperaturas mais elevadas, o que as torna como opções interessantes para indústria de produtos derivados de frutas.
\end{abstract}

Palavras-chave: Frutas tropicais; Compostos bioativos; Conservação de frutas; Análise sensorial.

\section{Abstract}

This study aimed to develop and characterize three jelly formulations, using strawberry and acerola in an isolated and mixed form. Three formulations were developed: GA - acerola jelly; GM - strawberry jam; GAM - mixed acerola and strawberry jam. For the characterization of the samples, analyzes were carried out to determine $\mathrm{pH}$, ${ }^{\circ} \mathrm{Brix}$, total titratable acidity (TT), viscosity, moisture, total sugars (TA), glucose, sucrose, antioxidant activity (FRAP and ABTS) and total content of phenolics and flavonoids. In addition, the samples were submitted to a sensory acceptance test with 100 untrained tasters to verify their opinion in relation to the attributes appearance, color, aroma, flavor, consistency, global evaluation and purchase intention. The results showed that the jellies had soluble solids contents 
( ${ }^{\circ}$ Brix) ranging between 56-68.17 ${ }^{\circ}$ Brix, which were sufficient for gel formation. The jellies showed no significant difference $(\mathrm{p} \geq 0.05)$ only for the $\mathrm{pH}$, AT and sucrose parameters. The viscosity of the jellies decreased with increasing temperature. In this study it was noticed that the mixture of the two fruits increased the sensorial acceptance of the evaluated attributes; however, in terms of functionality and technological aspects, the jelly made only with acerola showed greater antioxidant activities and viscosity at higher temperatures, which makes them interesting options for the fruit-derived industry.

Keywords: Tropical fruits; Bioactive compounds; Conservation of fruits; Sensory analysis.

\begin{abstract}
Resumen
Este estudio tuvo como objetivo desarrollar y caracterizar tres formulaciones de gelatina, utilizando fresa y acerola en forma aislada y mixta. Se desarrollaron tres formulaciones: GA - jalea de acerola; GM - mermelada de fresa; GAM acerola mezclado y gelatina de fresa. Para la caracterización de las muestras se realizaron análisis para determinar pH, - Brix, acidez total titulable (TT), viscosidad, humedad, azúcares totales (TA), glucosa, sacarosa, actividad antioxidante (FRAP y ABTS) y el contenido total de fenólicos y flavonoides. Además, las muestras fueron sometidas a una prueba de aceptación sensorial con 100 catadores no capacitados para verificar su opinión en relación a los atributos apariencia, color, aroma, sabor, consistencia, evaluación global e intención de compra. Los resultados mostraron que las gelatinas tenían contenidos de sólidos solubles ( ${ }^{\circ}$ Brix) que oscilaban entre $56-68,17^{\circ}$ Brix, que eran suficientes para la formación de gel. Las medusas no mostraron diferencia significativa ( $\mathrm{p} \geq 0.05)$ solo para los parámetros de $\mathrm{pH}$, AT y sacarosa. La viscosidad de las gelatinas disminuyó al aumentar la temperatura. En este estudio se notó que la mezcla de los dos frutos incrementó la aceptación sensorial de los atributos evaluados; sin embargo, en términos de funcionalidad y aspectos tecnológicos, la gelatina elaborada solo con acerola mostró mayor actividad antioxidante y viscosidad a temperaturas más altas, lo que las convierte en opciones interesantes para la industria derivada de frutas.
\end{abstract}

Palabras clave: Frutas tropicales; Compuestos bioactivos; Conservación de frutos; Análisis sensorial.

\title{
1. Introdução
}

Inúmeras frutas in natura são desperdiçadas por não estarem sensorialmente perfeitas, ainda que em boas condições de consumo. As transformações destas matrizes em outros produtos através de técnicas adequadas passam a ser um meio de reduzir desperdícios. O aproveitamento de frutas na elaboração de geleias pode ampliar o consumo, especialmente das frutas que não estão em ótima qualidade sensorial (Oliveira et al., 2018).

As frutas são consideradas as principais fontes de vitaminas e compostos bioativos, que estão associados à redução do risco de doenças cardiovasculares e neurodegenerativas (Aboul-enein et al., 2013). Dentre os compostos bioativos, destacamse os carotenoides e compostos fenólicos, os quais têm sido associados à capacidade antioxidante de frutas (Eberhardt et al., 2000; Haminiuk et al., 2012; Vidigal et al., 2011), propriedade funcional que está realacionada a presença de um anel aromático com um ou mais grupos hidroxilas (Tiveron, 2010).

O morango (Fragaria L.) é uma importante fonte de fibras, minerais e vitaminas os quais vem sendo associados a benefícios à saúde. Contudo, o conteúdo destes nutrientes pode variar de acordo com a cultivar (Antunes et al., 2016). Alguns estudos demonstraram que o morango possui importantes atividades bioativas, como ação antioxidante, anti-inflamatória e a anti-hipertensiva (Giampieri et al., 2014). Já a acerola (Malpighia punicifolia L.) é bastante reconhecida por seu elevado teor de vitamina $\mathrm{C}$, além de vitamina A, tiamina, riboflavina, niacina, proteínas e sais minerais. Sua capacidade antioxidante está relacionada, sobretudo, á presença de flavonoides e vitamina C (Correia et al., 2012). Tem como característica possuir polpa suculenta e casca protetora que, ao amadurecer, muda a coloração de verde para amarelo-avermelhado e finalmente para vermelho ou roxo, quando completamente amadurecida, dependendo da variedade. A mudança de pigmento está ligada a mudanças bioquímicas complexas que envolvem todos os seus principais compostos (Malegori et al., 2017).

Uma alternativa de utilização destes frutos, como forma de prolongar sua vida útil, devido a sua alta perecibilidade, prolongar as propriedades bioativas é o processamento na forma de diferentes produtos, tais como polpas, sucos e geleias. De acordo com regulamento técnico que consta na Resolução $n^{\circ} 272$, geleia é definida como o produto obtido pela cocção de frutas, inteiras ou em pedaços, polpa ou suco de frutas, com açúcar e água, e concentrado até consistência gelatinosa (Anvisa, 
2005). Segundo a legislação brasileira de geleias de frutas, representada pela Resolução $\mathrm{n}^{\circ} 12$ de 24 de julho de 1978 e Resolução Normativa $\mathrm{n}^{\circ} 15$ de 4 de maio de 1978, esse produto pode ser classificado como geleia extra, geleia simples e geleia premium. As geleias comuns e extras ainda podem ser classificadas como "simples" (termo não obrigatório), quando preparadas com um único tipo de vegetal, ou "mistas" (termo facultativo) quando elaborada com duas ou mais espécies vegetais. As geleias mistas são alternativas importantes, pois unem características nutricionais de duas ou mais frutas, além de proporcionar agradáveis características sensoriais, e vêm conquistando, gradativamente, espaço nobre no mercado consumidor (Zotarelli et al., 2008).

O morango e a acerola por serem frutos perecíveis, necessitam de alternativas de processamento que prolonguem a vida útil do produto, conservando as suas propriedades nutricionais, funcionais e sensoriais. Desta forma, considerando as informações supracitadas, objetiva-se neste estudo analisar as propriedades tecnológicas, físico-químicas, antioxidantes e sensoriais de geleias de acerola e/ou morango.

\section{Metodologia}

\subsection{Matéria prima e preparação das geleias de frutas}

Todos os ingredientes foram obtidos na cidade de Cuité/PB. As frutas maduras foram adquiridos na feira livre da referida cidade, sendo transportados para o laboratório em caixas de isopor.

Para efeito comparativo, neste estudo foram elaborados 3 diferentes tipos de geleias, sendo: GA, geleia de acerola; GM, geleia de morango e GAM, geleia mista de morango e acerola, cujas quantidades de ingredientes estão descritas na Tabela 1.

Tabela 1 - Formulação das geleias de frutas tropicais.

\begin{tabular}{lccc}
\hline Ingredientes & Geleia de acerola (GA) & Geleia de morango (GM) & $\begin{array}{c}\text { Geleia mista de acerola e } \\
\text { morango } \\
\text { (GAM) }\end{array}$ \\
\hline Açúcar refinado (\%) & 40 & 40 & 40 \\
Polpa de acerola (\%) & 40 & - & 20 \\
Polpa de morango (\%) & - & 40 & 20 \\
Água (\%) & 20 & 20 & 20 \\
\hline
\end{tabular}

Fonte: Autores.

Para o processamento das geleias, as frutas foram previamente selecionadas, lavadas em água corrente e potável para a remoção de sujidades, sanitizadas em imersão com solução de hipoclorito de sódio a 150 ppm por 15 minutos. Os frutos foram triturados com água em liquidificador industrial Industrial Shop ${ }^{\circledR}$ (modelo 2 Litros Alta Rotação Industrial Inox 800w). A mistura resultante foi misturada com o açúcar nas proporções ilustradas na Tabela 1 e submetido à cocção em temperatura de $175 \pm 10{ }^{\circ} \mathrm{C}$ até alcançar o ponto de geleia (teor de sólidos solúveis [ ${ }^{\circ}$ Brix] entre 57-67\%).

\subsection{Caracterização das geleias}

As geleias processadas foram avaliadas, em triplicata, após 1 dia do processamento, quanto aos parâmetros tecnológicos, físicos, físico-químicos, de propriedades antioxidantes e aceitação sensorial, conforme metodologias descritas a seguir. 


\subsection{Propriedades tecnológicas, físicas e físico-químicas}

Após o processamento as geleias foram submetidas às análises de atividade de água $\left(\mathrm{a}_{\mathrm{a}}\right)$ a $25{ }^{\circ} \mathrm{C}$, usando aparelho Aqualab $^{\circledR}$ (modelo C2-2, Water Activity System ${ }^{\circledR}$, Washington-USA); sólidos solúveis totais - SST $\left({ }^{\circ}\right.$ Brix, g/100 g) em refratômetro digital (HI 96801, Hanna Instruments, São Paulo, Brazil) a $25 \pm 1{ }^{\circ} \mathrm{C}$; e pH, em potenciômetro digital (modelo Q400, Quimis ${ }^{\circledR}$, Diadema, São Paulo, Brasil) (AOAC, 2016). Para as medidas de viscosidade aparente, foi utilizado o viscosímetro Brookfield (modelo DV II+Pro) com spindle SC4-21, acoplado a um banho termostático para controlar a temperatura das amostras. As leituras de viscosidade aparente (mPa.s) das geleias foram feitas a $5,15,25$ e $35^{\circ} \mathrm{C}$ na velocidade de rotação de $60 \mathrm{rpm}$, utilizando-se $7,5 \mathrm{~mL}$ das amostras no porta amostra.

Ainda foram determinados acidez total titulável (ATT), umidade, cinzas, glicose, sacarose e açúcares totais (AT) de acordo com metodologia recomendada pela Association of Official Analytical Chemists (AOAC, 2016).

\subsection{Determinação do conteúdo total de compostos fenólicos, flavonoides e atividades antioxidantes}

\subsubsection{Preparação do extrato}

Para a obtenção do extrato, $1 \mathrm{~g}$ das geleias foi homogeneizado em $10 \mathrm{~mL}$ de metanol (Merck, Darmstadt, Alemanha) a 80\% por 10 min usando um mini-Turrax (TE-102, Tecnal, Piracicaba, São Paulo, Brasil); após homogeneização, os extratos foram mantidos em descanso por 24 horas e, posteriormente, filtrados com um papel de filtro de $125 \mathrm{~mm}$ (Whatman ${ }^{\circledR}$, GE Healthcare, Chicago, IL, USA).

\subsubsection{Conteúdo total de fenólicos e flavonoides}

O conteúdo de fenólicos totais foi analisado utilizando o método de Folin-Ciocalteu. Para tal, $250 \mu \mathrm{L}$ de cada extrato foram misturados em tubo de ensaio com $1250 \mu \mathrm{L}$ do reagente Folin-Ciocalteau a 10\%. As soluções foram agitadas em Vortex (Q220M, Quimis, Diadema, São Paulo, Brazil) e armazenadas em temperatura ambiente $\left(25 \pm 0,5{ }^{\circ} \mathrm{C}\right)$ na ausência da luz por 6 min. Após, foram adicionados $1 \mathrm{~mL}$ da solução de carbonato de sódio a 7,5\%. A mistura foi levada ao banho maria (Modelo BS-3030, Raypa, Barcelona, Espanha) a uma temperatura de $50 \pm 0,5^{\circ} \mathrm{C}$ durante 5 min. Após, a absorbância foi medida a 765 nm utilizando espectrofotômetro (BEL Photonics, Piracicaba, São Paulo, Brasil). Também foi realizado um branco com a ausência dos extratos para zerar o espectrofotômetro. O conteúdo de compostos fenólicos totais das amostras foi determinado utilizando uma curva padrão preparada com ácido gálico (Sigma-Aldrich, St. Louis, MA, USA). Os resultados foram expressos em mg equivalentes de ácido gálico (EAG) por 100 gramas de amostra (mg EAG/100 g) (LIU et al., 2002).

Ainda, determinou-se o teor total de flavonoides, segundo metodologia descrita por Guevara-Figueroa et al. (2010). Uma alíquota de 0,5 mL do extrato foi adicionada a $2 \mathrm{~mL}$ de água destilada em um tubo de ensaio. Em seguida, adicionaramse $150 \mu \mathrm{L}$ de nitrito de sódio a $5 \%$. Após $5 \mathrm{~min}, 150 \mu \mathrm{L}$ de cloreto de alumínio a $10 \%$ foram adicionados e, após $6 \mathrm{~min}, 1 \mathrm{~mL}$ de hidróxido de sódio a $1 \mathrm{M}$, seguido pela adição de $1,2 \mathrm{~mL}$ de água destilada. A absorbância da amostra foi medida a 510 nm usando um espectrofotômetro (BEL Photonics) contra um branco na ausência dos extratos. O teor de flavonoides totais foi determinado usando uma curva padrão de equivalentes de catequina (EC) (Sigma-Aldrich). Os resultados foram expressos em mg equivalentes de catequina (EC) por 100 gramas de amostra (mg EC/100 g).

\subsubsection{Atividade antioxidante pelo método do radical ABTS}

A capacidade dos extratos das amostras de geleia em capturar o cátion ABTS $^{\bullet+}$ (2,2-azino-bis (3-etilbenzo-tiazoline)6-sulfonic acid) foi determinada pelo método $\mathrm{ABTS}^{\cdot+}$. Inicialmente, preparou-se o reagente $\mathrm{ABTS}^{\bullet+}$, que é obtido pela reação de $5 \mathrm{~mL}$ de ABTS a $7 \mathrm{mM}$ com $88 \mu \mathrm{L}$ de persulfato de potássio a $140 \mathrm{mM}$ (concentração final de 2,45 mM), segundo método 
de Sariburun et al. (2010). Posteriormente, manteve-se em repouso, a temperatura ambiente de 12 a 16 horas em ausência de luz, para formação do $\mathrm{ABTS}^{\bullet+}$. Uma vez formado, o radical foi diluído com água destilada até obter valor de absorbância de 0,800 - 0,900 a $734 \mathrm{~nm}$. A leitura da absorbância foi feita a $734 \mathrm{~nm}$ em espectrofotômetro (BEL Photonics), com a mistura de reação $(600 \mu \mathrm{L})$ contendo $100 \mu \mathrm{L}$ da amostra e $500 \mu \mathrm{L}$ de solução ABTS ${ }^{\bullet+}$. Também foi feita uma solução "controle" que consistiu em uma alíquota de $100 \mu \mathrm{L}$ do solvente extrator dos extratos adicionada de $500 \mu \mathrm{L}$ do radical ABTS ${ }^{+}$. A solução "branca" foi o solvente extrator de cada extrato, utilizada para zerar o espectrofotômetro. Como referência, foi utilizado o Trolox e os resultados foram expressos em $\mu \mathrm{mol}$ de capacidade antioxidante equivalente ao trolox por $\mathrm{g}$ da amostra $(\mu \mathrm{mol}$ CAET/g).

\subsubsection{Atividade antioxidante pela capacidade redutora de ferro - FRAP}

A capacidade dos extratos das amostras de geleia em reduzir o íon férrico $\left(\mathrm{Fe}^{3+}\right)$ a íon ferroso $\left(\mathrm{Fe}^{2+}\right)$ foi determinada pelo método FRAP, adaptado segundo Rockenbach et al. (2011). Inicialmente, preparou-se o reagente FRAP com solução tampão acetato de sódio anidro 0,3 M (pH 3,6), TPTZ (2,4,6-tris (2-pyridyl)-s-triazine) a $10 \mathrm{mM} / \mathrm{L}$ em uma solução de HCl a $40 \mathrm{mM}$ e cloreto férrico $\left(\mathrm{FeCl}_{3}\right)$ a $20 \mathrm{mM}$. Em uma alíquota de $200 \mu \mathrm{L}$ do extrato foram adicionados $1800 \mu \mathrm{L}$ da solução FRAP. Em seguida, os tubos contendo o extrato e a solução FRAP foram agitados e levados ao banho-maria a $37{ }^{\circ} \mathrm{C}$ por 30 min. A absorbância foi medida a $593 \mathrm{~nm}$ em espectrofotômetro (BEL Photonics). A curva padrão foi realizada com Trolox 1 $\mathrm{mM}$ e os resultados expressos em $\mu \mathrm{mol}$ capacidade antioxidante equivalente ao Trolox por g da amostra ( $\mu \mathrm{mol}$ CAET/g).

\subsection{Análise sensorial}

A pesquisa foi realizada com 100 colaboradores não treinados, representados por estudantes e servidores da Universidade Federal de Campina Grande, UFCG, Campus de Cuité, Paraíba, Brasil. O painel foi selecionado com base nos hábitos e interesse em consumir geleias de frutas, constituído tanto pelo gênero feminino como masculino, com faixa etária variando de 18 a 45 anos de idade, não apresentando nenhum problema de saúde ou deficiência física que viesse a comprometer a avaliação sensorial dos produtos.

Os consumidores avaliaram sensorialmente as três formulações de geleias com relação à aceitação sensorial dos atributos aparência, cor, aroma, sabor, consistência e avaliação global utilizando escala hedônica estruturada de nove pontos (1 = desgostei extremamente $;$ = nem gostei/nem desgostei; 9 = gostei extremamente). Os avaliadores foram orientados a marcar a opção que melhor se adequava ao produto, em relação aos atributos avaliados (Stone \& Sidel, 1993). Paralelamente, também foi avaliada a intenção de compra, empregado uma escala hedônica estruturada de cinco pontos $(1=$ certamente não compraria; 3 = talvez comprasse/talvez não comprasse; 5 = certamente compraria).

Em ambos os testes, as amostras foram padronizadas e servidas, simultaneamente e de forma aleatória, a temperatura ambiente $\left(25 \pm 0,5^{\circ} \mathrm{C}\right)$, em copos de plásticos de cor branca codificados com números aleatórios de 3 dígitos. Juntamente com as amostras foram oferecidos aos provadores bolacha e água e estes foram orientados a entre uma amostra e outra fazer o uso da bolacha e da água, para remoção do sabor residual, e a provarem estas da esquerda para direita. Os testes foram realizados em cabines individuais utilizando-se luz branca, longe de ruídos e odores, em horários que não compreendessem uma hora antes e duas horas após o almoço.

\subsection{Análise Estatística}

Os resultados das análises tecnológicas, físico-químicas, propriedades antioxidantes e sensoriais foram submetidos à Análise de Variância (ANOVA), seguido do teste de Tukey, ao nível de significância de 5\%, para comparação das médias. 
Para o cálculo destes dados, utilizou-se o software Statistica, versão 13 (StatSoft). Os gráficos foram plotados utilizando o software Matlab.

\section{Resultados e Discussão}

\subsection{Propriedades tecnológicas, físicas e físico-químicas}

As propriedades tecnológicas e físicas das geleias estão apresentadas na Tabela 2. A geleia de morango (GM) apresentou maior teor de sólidos solúveis totais ( ${ }^{\circ}$ Brix), seguida da geleia mista (GM), quando comparadas formulações geleia de acerola (GA) (p < 0,05). Carneiro et al. (2012) analisaram geleias simples de morango e pêssego e encontraram para a geleia de morango teor de sólidos solúveis de $65^{\circ}$ Brix, valor semelhante aos encontrados neste trabalho para formulação de geleia mista. Já Ferreira (2013) avaliando geleia de morango encontrou valor menor que o determinado em GM, no estudo em tela, correspondente a $61,94{ }^{\circ}$ Brix.

Tabela 2 - Propriedades tecnológicas e físicas das geleias de frutas tropicais.

\begin{tabular}{ccccc}
\hline Geleias & $\mathbf{A}_{\mathbf{a}}$ & $\mathbf{p H}$ & $\begin{array}{c}\text { Acidez } \\
(\mathbf{g} / \mathbf{1 0 0} \mathbf{g})\end{array}$ & $\begin{array}{c}{ }^{\mathbf{B}} \mathbf{B r i x} \\
(\mathbf{g} / \mathbf{1 0 0} \mathbf{g})\end{array}$ \\
\hline GA & $0,90 \pm 0,00^{\mathrm{a}}$ & $3,43 \pm 0,06^{\mathrm{a}}$ & $0,98 \pm 0,01^{\mathrm{c}}$ & $56,00 \pm 0,00^{\mathrm{c}}$ \\
GM & $0,81 \pm 0,00^{\mathrm{c}}$ & $3,40 \pm 0,00^{\mathrm{a}}$ & $1,04 \pm 0,03^{\mathrm{b}}$ & $68,17 \pm 0,29^{\mathrm{a}}$ \\
GAM & $0,86 \pm 0,00^{\mathrm{b}}$ & $3,33 \pm 0,06^{\mathrm{a}}$ & $1,17 \pm 0,01^{\mathrm{a}}$ & $65,00 \pm 0,00^{\mathrm{b}}$ \\
\hline
\end{tabular}

GA - geleia de acerola; GM - geleia de morango; GAM - geleia mista de acerola e morango; $a_{a}$ - Atividade de água.

${ }^{\mathrm{abc} C}$ Comparação entre formulações. Valores na mesma coluna assinalados com letras sobrescritas diferentes são estatisticamente diferentes segundo o teste de Tukey $(\mathrm{p}<0.05)$.

Fonte: Autores.

A geleia de acerola apresentou maior valor $(\mathrm{p}<0,05)$ de atividade de água quando comparada ás demais formulações. No geral, á atividade de água detectada nas formulações de geléia variou entre 0,81 a 0,90, valores estes superiores aos determinados por Ruaro (2015) em geleia de abacaxi com adição de mucilagem de chia (0,62 a 0,64). Ferreira (2013) analisou a composição de geleias de morango e encontrou valor de atividade de água de 0,85 para geleia adoçada com açúcar e de 0,96 para geleia diet, valores próximos aos encontrados no presente estudo.

Não houve diferença estatística para o pH entre as formulações ( $\mathrm{p} \geq 0,05)$. Segundo Ribeiro et al. (2016), a acidez está relacionada com a presença de substâncias ácidas naturalmente contidas em frutas. Esses compostos, em especial o ácido cítrico, auxiliam no desenvolvimento da textura adequada das geleias, sendo um importante parâmetro físico-químico no controle de qualidade de geleias. Além disso, de acordo com Gava et al. (2008), valores de pH abaixo de 3,2 podem dificultar a formação de gel, resultando em geleias com baixa viscosidade e com aspecto amolecido. No presente estudo, os valores determinados para pH foram maiores que 3,2, justificando a consistência de gel alcançada após processamento. Paiva et al. (2015), ao avaliarem a qualidade de geleia de acerola e melão, observaram pH em torno de 3,3, o qual se equipara ao pH das geleias avaliada no presente estudo. Souza et al. (2021), ao avaliarem geleia de abacaxi com diferentes concentrações de pimenta, identificam valores de $\mathrm{pH}$ em torno de 4,5, valores superiores aos encontrados no presente estudo.

As geleias obtidas neste estudo apresentaram teores de acidez com variação de 0,98 a 1,17 g/100 g. Oliveira et al. (2016) formularam geleias de laranja com e sem hortelã, e encontraram valores inferiores aos identificados nesta pesquisa de 0,51 e $0,50 \mathrm{~g} / 100 \mathrm{~g}$, respectivamente. Nascimento et al. (2020) ao avaliarem geleia mista de uva e maracujá produzida a partir de formulações tradicionais, light e diet encontram valores de acidez que variaram entre 0,75 a 1,10 g/100 g valores inferiores 
aos encontrados nesta pesquisa. A acidez total está associada á presença de ácidos orgânicos, como o ácido cítrico e o ácido málico, sendo que a acidez influencia o sabor. Já o pH torna-se importante no que se refere á segurança dos alimentos, oferecendo uma indicação do grau de deterioração do alimento (Almeida, 2009). Sendo assim, baseando no pH mínimo para multiplicação e produção da toxina do Clostridium botulinum $(4,5)$ e o mínimo para a proliferação da maioria das bactérias $(4,0)$ (Franco \& Landgraf, 2005), podemos afirmar que a geleias de acerola e ou morango propostas não são propícias para o desenvolvimento destes micro-organismos.

Com os dados coletados no presente estudo podemos afirmar que, as geleias tradicionais mistas de morango e acerola apresentaram pH e teor de acidez ideais para formação do gel, pois não foi observado rompimento e nem dificuldade de formação do mesmo.

A viscosidade é um atributo de grande importância em produtos alimentícios que pode determinar sua aceitação ou não por parte do consumidor, bem como sua qualidade tecnológica. Na Figura 1 podemos ver o comportamento da viscosidade aparente das geleias em diferentes temperaturas submetida a uma agitação de $60 \mathrm{rpm}$. Viu-se que houve redução da viscosidade, com aumento da temperatura para todas as formulações. GA apresentou os maiores valores de viscosidade nas temperaturas de 5,15 e $25{ }^{\circ} \mathrm{C}$. Já GM e GAM apresentaram valores de viscosidade semelhante nas temperaturas 15 e $25{ }^{\circ} \mathrm{C}$. Na temperatura de $35^{\circ} \mathrm{C}$ as geleias apresentaram valores de viscosidade aproximadas.

Figura 1 - Viscosidade aparente (mPa.s) das geleias de acerola, morango e mista em diferentes temperaturas.

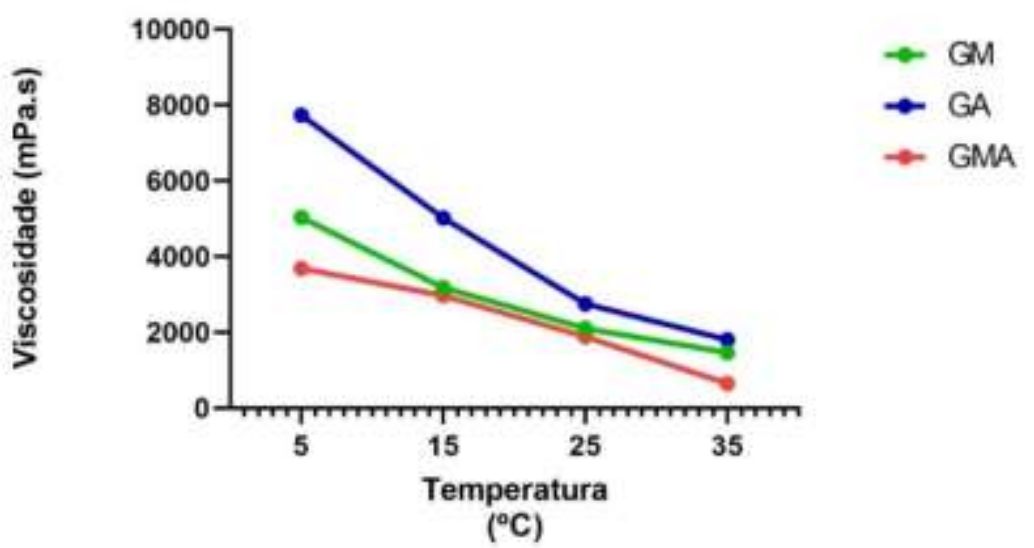

Fonte: Autores.

A composição físico-química das diferentes formulações de geleias são descritas na Tabela 3. A geleia GA apresentou maior teor de umidade $(\mathrm{p}<0,05)$, justificando a maior atividade de água determinada nesta formulação (Tabela 2). Em contrapartida, GM apresentou o menor teor de umidade $(\mathrm{p}<0,05)$. Essas diferenças entre as formulações podem ser explicadas pelas diferenças existentes nas composições das frutas, como a maior ou menor proporção de fibras e outros constituintes. As geleias possuem um teor de umidade relativamente alto por se tratar de um produto com grande quantidade de água na sua formulação. A legislação atual brasileira, Resolução n. 272, 2005, não estabelece um valor limite para a umidade de geleia de frutas (Brasil, 2005). 
Tabela 3 - Propriedades físico-químicas das geleias de frutas tropicais.

\begin{tabular}{cccccc}
\hline Geleias & $\begin{array}{c}\text { Umidade } \\
(\mathbf{g} / \mathbf{1 0 0} \mathbf{g})\end{array}$ & $\begin{array}{c}\text { Cinzas }(\mathbf{g} / \mathbf{1 0 0} \\
\mathbf{g})\end{array}$ & $\begin{array}{c}\text { Açúcares Totais } \\
(\mathbf{g} / \mathbf{1 0 0} \mathbf{g})\end{array}$ & $\begin{array}{c}\text { Glicose }(\mathbf{g} / \mathbf{1 0 0} \\
\mathbf{g})\end{array}$ & $\begin{array}{c}\text { Sacarose }(\mathrm{g} / \mathbf{1 0 0} \\
\mathbf{g})\end{array}$ \\
\hline GA & $41,81 \pm 0,15^{\mathrm{a}}$ & $0,16 \pm 0,02^{\mathrm{b}}$ & $61,42 \pm 0,65^{\mathrm{a}}$ & $9,27 \pm 0,10^{\mathrm{b}}$ & $49,54 \pm 0,52^{\mathrm{a}}$ \\
GM & $28,99 \pm 0,15^{\mathrm{c}}$ & $0,20 \pm 0,02^{\mathrm{ab}}$ & $61,98 \pm 0,12^{\mathrm{a}}$ & $9,44 \pm 0,02^{\mathrm{a}}$ & $49,92 \pm 0,10^{\mathrm{a}}$ \\
GAM & $32,06 \pm 0,19^{\mathrm{b}}$ & $0,23 \pm 0,00^{\mathrm{a}}$ & $62,03 \pm 0,07^{\mathrm{a}}$ & $9,38 \pm 0,03^{\mathrm{ab}}$ & $50,02 \pm 0,07^{\mathrm{a}}$ \\
\hline
\end{tabular}

GA - geleia de acerola; GM - geleia de morango; GAM - geleia mista de acerola e morango.

${ }^{\mathrm{abc} C}$ Comparação entre formulações. Valores na mesma coluna assinalados com letras sobrescritas diferentes são estatisticamente diferentes segundo o teste de Tukey $(\mathrm{p}<0,05)$.

Fonte: Autores.

Em se tratando dos teores de cinzas, GA apresentou menores $(\mathrm{p}<0,05)$ teores de cinzas $(0,16 \pm 0,02 \mathrm{~g} / 100 \mathrm{~g})$, quando comparada às demais formulações. Já em GMA foram verificados os maiores teores de cinzas (p < 0,05), podendo ser justificado pela combinação de frutas em sua formulação. Dessimoni-Pinto et al. (2011), ao elaborarem geleias a partir de polpa de jabuticaba, identificaram $0,09 \mathrm{~g} / 100 \mathrm{~g}$ de cinzas, enquanto a geleia constituída de $100 \%$ jabuticaba com adição de cascas apresentou valor bem superior, $0,66 \%$. O teor de cinzas depende do tipo de solo em que a frutífera for cultivada e da composição do mesmo (Khan et al., 2014).

Não houve diferença estatística entre as geleias para os parâmetros açúcares totais e sacarose $(\mathrm{p} \geq 0,05)$, o que já era esperado tendo em vista que as formulações continham as mesmas concentrações de açúcar. Dentre os dois açúcares determinados, a sacarose foi o açúcar majoritário em todas as formulações. O teor de glicose foi maior na formulação GM $(9,44 \pm 0,02 \mathrm{~g} / 100 \mathrm{~g})$, seguido da geleia GAM $(9,38 \pm 0,03 \mathrm{~g} / 100 \mathrm{~g})$, possivelmente realcionado ao teor de glicose do morango.

\subsection{Determinação do conteúdo total de compostos fenólicos, flavonoides e atividades antioxidantes}

$\mathrm{Na}$ Tabela 4 encontram-se os valores das propriedades antioxidantes das geleias.

Tabela 4 - Propriedades antioxidantes das geleias de frutas tropicais.

\begin{tabular}{|c|c|c|c|c|}
\hline Geleias & $\begin{array}{c}\text { Fenólicos Totais (mg } \\
\text { EAG/100 g) }\end{array}$ & $\begin{array}{c}\text { Flavonoides Totais (mg } \\
\text { EC/100 g) }\end{array}$ & $\begin{array}{c}\text { FRAP }(\mu \mathrm{mol} \\
\text { CAET/g) }\end{array}$ & $\begin{array}{c}\text { ABTS }(\mu \mathrm{mol} \\
\text { CAET/g) }\end{array}$ \\
\hline GA & $295,28 \pm 0,01^{\mathrm{a}}$ & $58,13 \pm 0,01^{\mathrm{a}}$ & $1,64 \pm 0,01^{\mathrm{a}}$ & $12,00 \pm 0,01^{\mathrm{a}}$ \\
\hline GM & $295,28 \pm 0,01^{\mathrm{a}}$ & $58,14 \pm 0,01^{\mathrm{a}}$ & $0,72 \pm 0,01^{\mathrm{c}}$ & $10,61 \pm 0,01^{\mathrm{b}}$ \\
\hline GAM & $106,60 \pm 0,01^{b}$ & $51,88 \pm 0,01^{b}$ & $0,76 \pm 0,01^{\mathrm{b}}$ & $9,89 \pm 0,01^{\mathrm{c}}$ \\
\hline
\end{tabular}

GA - geleia de acerola; GM - geleia de morango; GAM - geleia mista de acerola e morango; EAG - equivalentes de ácido gálico; EC = equivalentes de catequina; CAET - capacidade antioxidante equivalente ao Trolox.

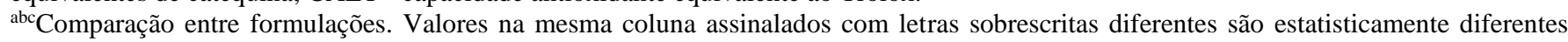
segundo o teste de Tukey $(\mathrm{p}<0,05)$.

Fonte: Autores.

A acerola é conhecida pelos seus altos níveis de ácido ascórbico, ácidos fenólicos, flavonoides, antocianinas e outros compostos importantes para a saúde humana, possui um poder antioxidante superior ao de outras frutas consideradas fontes de compostos fenólicos, como uva, açaí, morango, cereja e manga (Cruz et al., 2019). Todavia, parece que o uso da acerola no 
processamento da geleia não influenciou na permanência desta característica, considerando que tanto geleia de acerola quanto de morango apresentaram o mesmo conteúdo de compostos fenólicos totais $(\mathrm{p} \geq 0,05)$ (Tabela 4).

A geleia mista (GAM) apresentou um teor de fenólicos totais menor do que o das outras geleias ( $\mathrm{p}<0,05)$. É também importante citar que a concentração de compostos fenólicos na acerola e no morango depende fortemente do grau de maturação (Nóbrega et al., 2015) e da variedade dos frutos (Mariano-nasser et al., 2017). Estas diferenças na composição podem ser resultado tanto da variedade dos frutos, do local de cultivo, da temperatura, do tipo de solo, entre outros fatores do local de cultivo do morango e da acerola, fatores estes que podem alterar a composição dos frutos. Os teores de flavonoides em frutas podem variar até $100 \%$ entre um semestre e outro, sendo estes influenciados por fatores extrínsecos, tais como condições climáticas, época de plantio, colheita e grau de maturidade dos frutos (Araújo, 2017).

$\mathrm{Na}$ Tabela 4 também observa-se que GA apresentou menor teor de flavonoides totais quando comparada às outras geleias ( $\mathrm{p}<0,05)$. Mesmo assim, a atividade antioxidante determinada pelos métodos de FRAP e ABTS nessa formulação foi significativamente maior às demais geleias avaliadas $(\mathrm{p}<0,05)$, sendo possivelmente influenciado pelo maior conteúdo total de compostos fenólicos detectado $(295,28 \pm 0,01 \mathrm{mg}$ EAG/100 g) nesta amostra. A capacidade antioxidante segundo a metodologia de ABTS foi significativamente menor $(\mathrm{p}<0,05)$ para GAM $(9,89 \pm 0,01 \mu \mathrm{mol}$ CAET/g), que também apresentou menor teor de compostos fenólicos totais $(106,60 \pm 0,01 \mathrm{mg}$ EAG/100 g).

\subsection{Caracterização sensorial}

Na Tabela 5 são apresentados os resultados referentes aos testes de aceitação sensorial e intenção de compra das diferentes formulações de geleias.

Tabela 5 - Aceitação sensorial e intenção de compra das geleias.

\begin{tabular}{cccc}
\hline Atributos Sensoriais & GA & GM & GAM \\
\hline Aparência & $8,60 \pm 0,60^{\mathrm{a}}$ & $8,21 \pm 0,52^{\mathrm{b}}$ & $8,43 \pm 1,01^{\mathrm{ab}}$ \\
Cor & $8,11 \pm 0,71^{\mathrm{b}}$ & $8,16 \pm 0,56^{\mathrm{ab}}$ & $8,36 \pm 0,88^{\mathrm{a}}$ \\
Aroma & $8,14 \pm 0,57^{\mathrm{b}}$ & $8,29 \pm 0,54^{\mathrm{ab}}$ & $8,39 \pm 0,78^{\mathrm{a}}$ \\
Sabor & $8,24 \pm 0,57^{\mathrm{b}}$ & $8,29 \pm 0,54^{\mathrm{ab}}$ & $8,51 \pm 0,87^{\mathrm{a}}$ \\
Consistência & $8,02 \pm 0,65^{\mathrm{b}}$ & $8,41 \pm 0,55^{\mathrm{a}}$ & $8,34 \pm 0,93^{\mathrm{a}}$ \\
Avaliação global & $8,17 \pm 0,62^{\mathrm{ab}}$ & $7,97 \pm 0,81^{\mathrm{b}}$ & $4,28 \pm 0,84^{\mathrm{a}}$ \\
Intenção de compra & $4,02 \pm 0,89^{\mathrm{ab}}$ & $3,82 \pm 8,2^{\mathrm{b}}$ & \\
\hline
\end{tabular}

GA - geleia de acerola; GM - geleia de morango; GAM - geleia mista de acerola e morango.

${ }^{a b c}$ Comparação entre formulações. Valores na mesma linha assinalados com letras sobrescritas diferentes são estatisticamente diferentes segundo o teste de Tukey $(\mathrm{p}<0,05)$.

Fonte: Autores.

Para Cunha et al. (2010), a análise sensorial é um meio de traduzir a opinião e a intenção de compra do consumidor frente a determinado produto, em números, revelando-se assim, de grande importância a sua verificação para a real avaliação do potencial econômico do produto a ser oferecido à população.

De um modo geral, GAM recebeu as maiores pontuações para os atributos cor, aroma, sabor e consistência, quando comparada a geleia de acerola e maiores notas para avaliação global e intenção de compra, quando comparada á geleia de morango $(\mathrm{p}<0,05)$, mostrando que a mistura das duas frutas potencializa essas características sensoriais numa geleia. Mesmo com as diferenças estatísticas, todas as formulações receberam notas que variaram entre os termos hedônicos "gostei muito" a "gostei muitíssimo", o que pode ter influenciado nas intenções de compras, cujos termos hedônicos estiveram entre 
"possivelmente compraria" a "compraria".

A partir das médias das notas atribuídas para as geleias, percebe-se que no quesito aroma a maior nota foi 8,39 para a formulação GMA, valor maior do que o encontrado por Conceição et al. (2012) ao elaborar geleia mista de acerola com goiaba $(7,82)$, reforçando novamente a alta aceitabilidade das geleias elaboradas no presente trabalho.

\section{Conclusão}

As polpas de morango e acerola podem ser utilizadas na produção de geleias tradicionais de forma isolada ou combinada, devido ao bom desempenho das suas propriedades funcionais e tecnológicas, além das características sensoriais que garantem um atrativo ao consumidor. Todas as formulações apresentadas são uma excelente forma de conservar a fruta, por apresentar elevada acidez, baixo $\mathrm{pH}$ e reduzido teor de água, o que garante uma geleia de qualidade com as características físicas-químicas de acordo com a legislação, com viabilidade para produção em nível industrial. A partir das características funcionais tecnológicas e nutricionais pode-se inferir que todas as formulações têm grande potencial de comercialização, além de sugerirem potencial benefício a saúde do consumidor, devido às propriedades antioxidantes determinadas, se tornando assim um produto atrativo dentro do mercado de subprodutos e conservação de frutas perecíveis. A mistura das duas frutas aumentou a aceitação sensoriais dos atributos avaliados; todavia, em termos de funcionalidade e aspectos tecnológicos, a geleia feita apenas com acerola apresentou maiores atividades antioxidantes e viscosidade em temperaturas mais elevadas, o que as torna como opções interessantes para indústria de produtos derivados de frutas.

\section{Referências}

Aboul- Enein, H. Y., Berczynski, D. X. \& Kruk, I. (2013). Phenolic compounds: the role of redox regulation in neurodegenerative disease and cancer. Mini Reviews in Medicinal Chemistry, 13(3), 385-398.

Almeida, A. S. (2009). Qualidade, compostos bioativos e atividade antioxidante total de pedúnculos de cajueiros e frutos de umbuzeiros nativos do semi-árido do Piauí [Tese de Doutorado, Universidade Federal Rural do Semi-Árido do Rio Grande do Norte].

Antunes, L. E. C., Reisser Junior, C., \& Schwengber, J. E. (2016). Morangueiro. Embrapa Clima Temperado (CPACT).

Anvisa. (2005). Resolução RDC $n^{\circ}$ 272. Aprova o "Regulamento Técnico para produtos de vegetais, produtos de frutas e cogumelos comestíveis". Ministério da Saúde. https://bvsms.saude.gov.br/bvs/saudelegis/anvisa/2005/rdc0272_22_09_2005.html

Araújo, I. M. C. de. (2017). Caracterização bioativa de resíduos de frutas tropicais [Trabalho de Conclusão de Curso, Universidade Federal do Rio Grande do Norte].

Association Of Official Analytical Chemists - International (AOAC). (2016). Official Methods of Analysis (18a ed). AOAC.

Brasil. Ministério da Saúde. Secretaria de Vigilância Sanitária. (2005). Resolução RDC $N^{o} 272$. Regulamento Técnico Para Produtos de Vegetais, Produtos de Frutas e Cogumelos Comestíveis.

Carneiro, A. P. G., Costa, E. A., Soares, D. J., Moura, S. M. \& Constant, P. B. L. (2012). Caracterização fisico-quimica dos frutos in natura e geleias de morango e pêssego, e aspectos de rotulagem do produto ao consumidor. Revista Brasileira de Produtos Agroindustriais, 14 (3), $295-298$.

Conceição, A. L. S, Cedraz, A. K, Santos, C. C, Silva, M. S, Cardoso, R. L. (2012). Elaboração e Caracterização Química, Fisico Química e Sensorial de Geleia Mista de Acerola com Goiaba. Enciclopédia Biosfera. Centro Científico Conhecer, 8(15), 832.

Correia, R. T. P., Borges, K. C., Medeiros, M. F. \& Genovese, M. I. (2012). Bioactive compounds and phenolic-linked functionality of powdered tropical fruit residues. Food Science and Technology International, 18(6), 539-547.

Cunha, M. A. A., Andrade, A. C. W., Ferminani, A. F., Appelt, P. \& Buratto, A. P. (2010). Barras alimentícias formuladas com resíduos de soja. Revista Brasileira de Pesquisa em Alimentos, 1(2), 89-96.

Cruz, R. G. da., Beney, L., Gervais, P., Lira, S. P. de., Vieira, T. M. F. S. \& Dupont, S. (2019). Comparison of the antioxidant property of acerola extracts with synthetic antioxidants using an in vivo method with yeasts. Food Chemistry, 277, 698-705.

Dessimoni-Pinto, N. A. V., Silva, V. M., Batista, A. G., Vieira, G., Souza, C. R., Dumont, P. V. \& Santos, G. K. M. (2010). Características físico-químicas da amêndoa de Macaúba e seu aproveitamento na elaboração de barras de cereais. Alim. Nutr., 21(1), 77-84.

Eberhardt, M., Lee, C. \& Liu, R. (2000). Antioxidant activity of fresh apples. Nature, 405, 903-904. https://doi.org/10.1038/35016151. 
Ferreira, C. Z. (2013). Composição de geleias de morango preparadas com açúcar, sucos de frutas ou edulcorantes [Trabalho de Conclusão de Curso, Universidade de Brasilia].

Franco, B. D. G. M. \& Landgraf, M. (2005). Microbiologia dos alimentos. Atheneu.

Gava, A. J., Silva, C. A. B. \& Frias, J. B.G. (2008). Tecnologia de alimentos: princípios e aplicações (2a ed). Nobel.

Giampieri, F., Alvarez-Suarez, J. M. \& Battino, M. (2014). Effects beyond antioxidant activity. Journal of Agricultural and Food Chemistry, 62(1), 38673876.

Guevara-Figueroa, T., Jiménez-Islas, H., Reyes, L., Mortensen, A., Laursen, B., Lin, L., León-Rodríguez, A. et al. (2010). Proximate composition, phenolic acids, and flavonoids characterization of commercial and wild nopal (Opuntia spp.). Journal of Food Composition and Analysis, $23,525-532$. 10.1016/j.jfca.2009.12.003.

Haminiuk, C. W. I. (2012). Phenolic compounds in fruits - an overview. International Journal of Food Science and Technology, 47(1), 2023-2044.

Khan, N., Shabbir, A., George, D., Hassan, G. \& Adkins, S. W. (2014). Plantas forrageiras supressivas como parte de um programa de manejo integrado para Parthenium hysterophorus L. Field Crops Res., 156, 172-179.

Liu, M., Li, X. Q., Weber, C., Lee, C. Y., Brown, J. \& Liu, R. H. (2002). Antioxidant and antiproliferative activities of raspberries. Journal of Agricultural and Food Chemistry, 50, 2926-2930.

Malegori, C., Marques, E. J. N., Freitas, S. T., Pimentel, M. F., Pasquini, C. \& Casiraghi, E. (2017). Comparing the analytical performances of Micro-NIR and FT-NIR spectrometers in the evaluation of acerola fruit quality, using PLS and SVM regression algorithms. Talanta, 165, $112-116$.

Mariano-Nasser, F. A. D., Nasser, M., Furlaneto, K. A., Ramos, J. A., Vieites, R. L. \& Pagliarini, M. K. V. (2017). Bioactive compounds in different acerola fruit cultivars. Semina: Ciências Agrárias, 38(4), 2505-2514.

Nascimento, T. A. et al. (2020). Caracterização química e sensorial de geleia mista de uva e maracujá nas formulações tradicional, light e diet. Research, Society and Development, 11(9), 1-20. https://doi.org/10.33448/rsd-v9i11.10026

Nóbrega, E. M., Oliveira, E. L., Genovese, M. I. \& Correia, R. T. P. (2015). The impact of hot air drying on the physical-chemical characteristics, bioactive compounds and antioxidant activity of acerola (Malphigia emarginata) residue. Journal of Food Processing and Preservation, $39,131-141$.

Oliveira, E. N. A., Feitosa, B. F. \& Souza, R. L. A. (2018). Tecnologia e processamento de frutas: doces, geleias e compotas. Editora IFRN. https://memoria.ifrn.edu.br/handle/1044/1664?show=full

Oliveira, M. M. T., Braga, T. R., Pinheiro, G. K., Silva, L. R., Vieira, C. B. \& Torres, L. B. V. (2016). Parâmetros físico-químicos, avaliação microbiológica e sensorial de geleias de laranja orgânica com adição de hortelã. Revista de la Facultad de Agronomía, 115(1), 29-34.

Paiva, C. A., Aroucha, E. M. M., Ferreira, R. M. A., Araújo, N. O. \& Silva, P. S. L. (2015). Alterações físico-químicas de geleias de melão e acerola durante o armazenamento. Revista Verde, 10(3), 18-23.

Ribeiro, L. M. P., Damasceno, K. A., Gonçalves, R. M. S., Gonçalves, C. A. A., Cunha, A. N. A. M. F. (2016). Acidez, sua relação com pH e qualidade de geleias e doces em barra. Boletim Técnico IFTM, (2), 14-19.

Rockenbach, I. I., Rodrigues, E., Gonzaga, L.V., Caliari, V., Genovesec, M. I., Gonçalves, A. E. S. S. \& Fett, R. (2011). Phenolic compounds content and antioxidant activity in pomace from selected red grapes (Vitis vinifera L. and Vitis labrusca L.) widely produced in Brazil. Food Chemistry, $127,174-179$.

Ruaro, T. T. (2015). Elaboração de geleia de abacaxi com adição de mucilagem de chia (Salvia hispânica) [Trabalho de Conclusão de Curso, Universidade Tecnológica Federal do Paraná].

Sariburun, E., Şahin, S., Demir, C., Türkben, C. \& Uylaşer, V. (2010). Phenolic content and antioxidant activity of raspberry and blackberry cultivars. Journal of Food Science, 75(4), 328-335. http://dx.doi. org/10.1111/j.1750-3841.2010.01571.x. PMid:20546390.

Stone, H. \& Sidel, J. (1993). Sensory evaluation practices. (2a ed.). Academic Press.

Souza, R. C. et al, (2021) Qualidade microbiológica, sensorial e físico-química de geleia de abacaxi com diferentes concentrações de pimenta. Research, Society and Development, 10(8), 1-7. https://doi.org/10.33448/rsd-v10i8.17718

Tiveron, A. P. (2010). Atividade antioxidante e composição fenólica de legumes e verduras consumidos no Brasil. [Dissertação de Mestrado, Universidade de São Paulo].

Vidigal, D. D., Dias, D. C. F. D., Dias, L. A. D. \& Finger, F. L. (2011). Changes in seed quality during fruit maturation of sweet pepper. Scientia Agricola, Piracicaba, 68(5), 535-539.

Zotarelli, M. F. et al. (2008). Avaliação de geléias mistas de goiaba e maracujá. Revista Ceres, 55(6), 562-567. 